\title{
End-to-End Networks Vs Named Data Network: A Critical Evaluation
}

\author{
Suhaidi Hassan ${ }^{a}$, Walid Elbreikia*, Mohamed Firdhous ${ }^{a, b}$, Adib M. Monzer Habbala \\ aInterNetWorks Research Lab, School of Computing, Universiti Utara Malaysia, 06010 UUM Sintok, Kedah, Malaysia \\ ${ }^{b}$ Faculty of Information Technology, University of Moratuwa, Moratuwa 10400, Sri Lanka \\ *Corresponding author: walid@internetworks.my
}

\section{Article history}

Received : 15 August 2014

Received in revised form:

15 October 2014

Accepted :15 November 2014

\section{Graphical abstract}

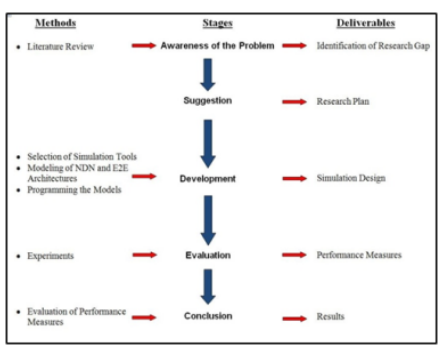

\section{Abstract}

Named data networking or information centric networking is the newest networking paradigm that gives foremost place to the contents in identification and dissemination. On the other hand, the end to end networking paradigm on which the Internet is currently built on places heavy emphasis on devices that make the architecture. The current Internet suffers from many shortcomings due to the misplaced emphasis. In order to overcome some of these deficiencies, researchers and developers have come up with patches and work around that have made the Internet more complex than it ought to be. Named data networking is a clean slate approach in building a network architecture overcoming all the current deficiencies and make it future safe. Several researchers have carried out comparative studies between named data networking and end to end networking. But these studies concentrate only on the features and capabilities of the networking paradigms. This is the first attempt at quantifying the performance the networking architectures experimentally. The authors in this paper present the results of the comparative study carried out experimentally in a simulated environment based on the final throughput. The results have been presented in a graphical form for easy visualization of results.

Keywords: Named data network; end-to-end network; performance measurement; throughput

(C) 2015 Penerbit UTM Press. All rights reserved.

\subsection{INTRODUCTION}

Named Data Networking (NDN) also known as Information Centric Network (ICN) is the new paradigm that envisages to move the Internet away from its current point to point architecture to a data centric one [1]. With NDN, the Internet will become data or content centric as opposed to its current host centric operation where communication is based on named hosts rather than the information stored in these hosts. In NDN more emphasis is placed on in-network caching for easy retrieval of contents and multi-party communication through replication and interaction models [2] (Ahlgren, Dannewitz, Imbrenda, Kutscher \& Ohlman, 2012). The innovation introduced by the NDN into the Internet would make it more efficient and resilient in the face of failures making it more suitable for present user requirements.

The advantages of NDN over the current Internet architecture includes reduced congestion, better delivery speeds, embedded security at data level itself and simpler device configuration. On the other hand the proposed architecture may have limitations in delivering certain types of data such as real time multimedia contents [3].

On the other hand the end-to-end networking paradigm on which the Internet is currently built on is based on identifying the nodes in which the data (Information) is stored [4]. The original architecture of the Internet was designed and built more than five decades ago and the fundamentals on which it was originally based on still remains intact with minor modifications carried out on an incremental basis for solving issues cropping up time to time [5]. The incremental solutions provided for addressing the issues have created many side effects. The downside of the endto-end networks in meeting the current user and application demands include lack of energy efficient communication, tight coupling of identification (address) to a particular geographical location, location ignorance of devices, inadequate support for seamless distributed services, strictly adhering to device-to-device communication rather than person-to-person communication, lack of in-built security, absence of separation between control, management and data planes, lack of isolation between applications sharing the same resources, non-support for asymmetric protocols and lack of quality of service guarantees [6]. In order to overcome these issues and face the requirements and challenges of the current and future demands require a cleanslate design approach for the Internet infrastructure. In this effort, several independent projects are being carried out throughout the world. Though the names of these projects and the finer details of proposed architectures differ from each other, they share many attributes including assumptions, objectives and architectural properties [2]. Details of the most prominent such projects are discussed in [5] Paul, et al. (2011).

In this paper, we compare the performance of named data network against that of end-to-end network based on a single attribute, throughput. Throughput has been selected as the metric of comparison as many other metrics such as latency, packet delivery ratio may be considered to be encompassed within this 
single metric. The experiments have been carried out in simulation environment setup using Network Simulator 3 (NS3) simulation tool.

This paper has been divided into five main sections as follows: Section 1 provides the introduction to the paper including the background information and problem addressed. Section 2 critically evaluates the related work carried out in this field with respect to their merits and demerits. Section 3 presents the methodology adopted in this study including the selection of measurement attributes. Section 4 describes the experimental setup used for testing the proposed mechanism along with an in depth analysis on the results. Finally Section 5 concludes the paper summarizing the findings.

\subsection{RELATED WORK}

This section takes an in depth look at the related work carried out by other researchers and published in journals, conference proceedings and technical reports. A critical analysis is carried out on the available literature with a view of understanding those works as well as identifying the research gap.

Jain (2006) [6] has presented a critical study on the shortcomings of the present Internet architecture with respect the demands of today's users and applications. In the same paper he highlights the features that must be included in a future network that would be equipped to meet the current as well as future demands. In the same paper he then discusses Generalized Inter Networking Architecture (GINA), a framework architecture that includes all the proposed features. The many proposed features are either identical or very similar to the ones that are expected to be in an NDN. Hence this paper can essentially be considered to be a comparison of features between E2E and NDN networks.

[8] Paul et al. (2011) have carried out a comprehensive survey of next generation internet projects carried out in many countries. At the beginning of the paper, they present an in depth analysis of the shortcomings of the current Internet architecture and the reasons that led these shortcomings. Then they discuss the features of different research efforts that have been carried out to develop the Future Internet. Hence this can also be considered as a comparison between the current and future Internet architectures based on their features.

[4] Blumenthal and Clark (2001) have discussed the end-toend arguments, a set of principles that had guided the design of the Internet. Then they show that how the emerging requirements could compromise the original design principles leading to the loss of some of the key features of the Internet including its ability to support new and unanticipated applications. In conclusion, they have taken an in depth look at the technical responses that had been put forward as a solution to the demands raised by the emerging trends. This paper stops short of discussing NDN or any other network with similar features. But, the ideas put forward by this paper way back in the beginning of the millennium can be considered as a serious effort towards establishing a whole new network paradigm away from the end-to-end networking principles.

[7] Oehlmann (2013) has compared the major components and operations carried out between NDN networks and E2E networks. He begins his comparison with the changes that would be introduced to the bottleneck of the hourglass shaped communication stack. In the E2E communication stack, IP layer that passes packets between devices sits in the neck, while in the NDN architecture, pieces of contents themselves make the bottleneck of the hourglass. In addition to almost all the other layers also see changes in terms of the functionality except the bottommost layer which provides the physical infrastructure. The
IP protocol that held the center stage in the E2E architecture is now pushed down in the NDN architecture occupying the layer just above the physical layer. Then he moves to the analysis of the types of packets used in both the network types. In E2E networks, there are a plethora of types of packets used for different purposes. But in NDN there are only two types of packets namely interest packets and data packets. This is huge reduction in complexity of packet types. On the operations carried in forwarding information from one node to the other, the author observes several similarities between the two network types. The naming structure used in NDN also shows similar characteristics with E2E. Both use hierarchical naming architecture except for the difference, in E2E the names are used to identifying devices and in NDN, the hierarchy represents the contents, content type and the owner (or holder) of the contents. In the area of routing, the intra domain routing is very much compatible with IP routing. Both are based on longest prefix matching lookups and becoming more detailed when getting closer to the contents. Finally the author shows that the information in NDN networks is self certifying making the data itself carry the security. On the other hand in E2E networks, security is implemented on hosts and network links leaving the data totally outside the security operations. This is a very interesting comparison as provides an insight into the technical aspects of both NDN and E2E networks.

[3] Melazzi and Chiariglione (2013) have listed the advantages of NDN (ICN) in a comprehensive manner. They have further stated that the content and service providers are compelled to patch current Internet to overcome to shortcomings and deficiencies. The patches introduced time to time in this manner include HTTP proxies, CDNs, multi-homing and intra domain multicasting etc. On the other hand, these functionalities are built in the NDN architecture. Thus this study presents an indirect comparison between these two networking paradigms.

[8] Kutscher, Flinck and Karl (2010) contrast the two network paradigms using the terms device centric for E2E and content centric for NDN. They further state that the device centric paradigm's main emphasis is on the interconnection of devices such as computers, mobile devices and routers. The real content such as web pages, video clips, or music files are transmitted over the Internet anonymously without clearly identifying the type of content or their importance. Thus the primary focus of the E2E network is devices and the information is given a lower priority from the network architecture perspective. On the contrary, NDN brings the content to the primary of the network architecture itself by giving it an identity and publicizing it irrespective of which device stores it. Also in the E2E paradigm, only the end devices can hold information. But in NDN the end devices as well as intermediary (networking) devices can hold information. This is commonly known as in network caching that enhances the dissemination of information. Hence this paper can be considered to be highlighting the differences between the two networking paradigms briefly.

[9] Siris, Ververidis, Polyzos and Liolis (2012) have discussed the integration of satellite and terrestrial links over an ICN networks. While discussing the features of these links that require special attention, they have highlighted the shortcomings of the present Internet architecture. The main reason highlighted by the authors for these inefficiencies is the patches and workarounds introduced time to time to meet the demands of users and emerging applications have become ossified into the Internet architecture. In the same paper, they highlighted how the features of the proposed NDN architecture could overcome these inefficiencies. Thus, this paper also provides a brief comparison between NDN and E2E architectures.

From the above discussion, it can be seen that the comparison between E2E and NDN architectures have been 
carried out only at the features level. So far no attempt has been made to compare the performance of these two paradigms experimentally. In this regards, this study can be considered as the first step towards filling this gap.

\subsection{METHODOLOGY}

The main objective of this study is to compare the performance of the named data network against end-to-end network through an extensive set of simulations. In order to achieve the said objective, it is critically important to follow a strict scientific methodology in order to make sure that the results obtained are valid and repeatable under similar conditions. The methodology used in this study has been adapted from the Design Science Research Methodology (DSRM) [10]. Figure 1 shows the research framework adopted in this study where the links between the stages in the DSRM research methodology, methods adopted in each stage and the main outcome (deliverable) at the completion of each stage. The vertical downward arrows show the main process flow while the horizontal arrows indicate the methods adopted at each stage and the expected outcomes of these methods.

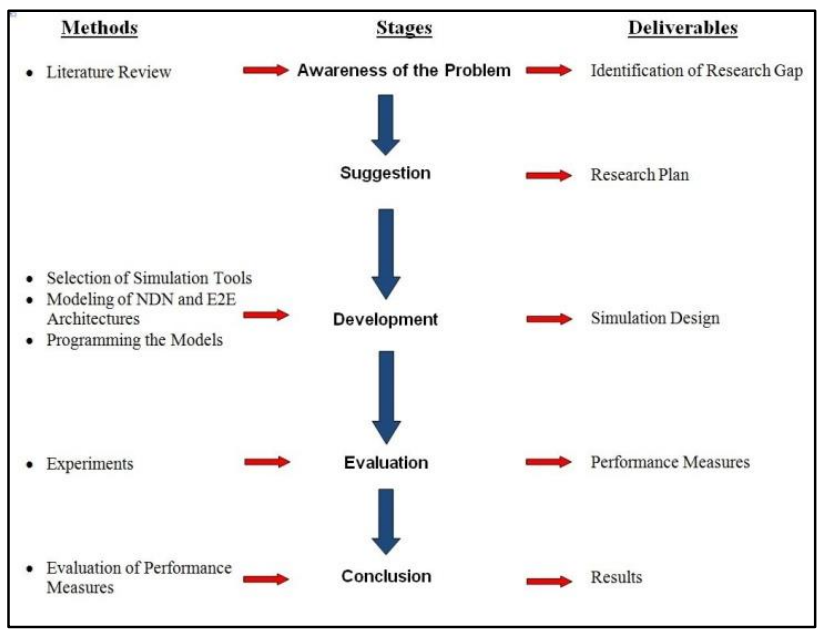

Figure 1 Research methodology

The proposed methodology consists of five main stages. They are namely awareness of the problem, suggestion, development, evaluation and conclusion. The details about the work carried out at each stage is explained below.

Awareness of the problem: During this stage, a thorough analysis of the related literature in the fields of NDN and E2E were carried out with the main objective of understanding these two networking paradigms and identifying an area to carry out an active investigation. The main outcome of this stage would be the research gap that needs to be filled at the end of this research.

Suggestion: During this stage, a detailed research plan based on the research gap identified in Stage 1 would be put forward. The research plan would include many important points including research focus and motivation, description of research problem along with the research questions, relevant areas to be consulted, research approach and final deliverables and contributions along with the limitations and future work.

Development: The heart of the research work is carried out During this stage. Initially a suitable simulation tool needs to be selected among the many possible tools available. It is necessary to understand the features, strengths and limitations of all these tools with respect to the selected research area need to be identified. After qualitatively evaluating many tools, it was decided to go for a generic simulation tool that can support both NDN and E2E architectures as opposed to specialized simulation tools that can support only specific network architectures. Finally Network Simulator version 3 commonly known as ns 3 was selected. The details of the reasons that led to the selection of ns3 will be discussed later. Then the network architecture (topology) needs to be decided and modeled in the simulator along with the necessary coding. Details of the model will be discussed in Section 4. The final outcome at the end of the development stage would be a complete simulation model ready for accepting inputs and process them.

Evaluation: During this stage, an extensive set of experiments will be carried out on the models developed in the previous stage. The experiments will designed to mimic all the possible practical scenarios in order to have comprehensive set of results that will help to compare the NDN and E2E architectures exhaustively based on the performance attribute selected. The outcome of this stage would be a comprehensive set of results representing the performance of the network models.

Conclusion: Finally the performance measures collected at the previous stage will be plotted and tabulated depending on the type of data for easy visualization and comparison. Based on the results of the comparison of performances, conclusions will be derived so as to which architecture performs better under which conditions.

\subsection{Selection of Simulator}

Simulation was the chose method for performance evaluation in this study as this technique is widely used in representing the dynamic behavior and responses of real systems [11]. Simulation enables analysis of a system, mechanism or protocol in a scalable, controllable and repeatable environments [12]. Also simulation is a cost effective method for testing various aspects of a system compared to other methods such as prototyping, or test bedding. Hence simulation has been selected in this study for analyzing the performance of NDN and E2E network architectures.

There are many discrete event network simulators available for use today. Some of these are commercial products requiring researchers to purchase them first and other are free and open source products that can be downloaded, used and modified free of cost. Some of the most popular tools that are available for communication network researchers are ns2, ns3, OPNET, OMNET, Qualnet, NetSim, J-Sim and GloMoSim [13]. Each one of these simulators has its strengths and weaknesses. There are many studies carried out on the capabilities and performance of simulators under various conditions and environments by several researchers. Due to the advantages and advanced features supported by ns 3 , it has been selected as the tool for running the experiments in this study $[14,15]$. A brief description of ns 3 is given in Sub Section 3.2.

\subsection{Network Simulator - Version 3 (ns3)}

Network Simulator 3 (ns3) is a free and open source network simulator that has been made available for teaching, research and development work under the GNU GPLv2 license [13]. Prior to the development of ns3, there were two more versions in the name of ns1 and ns2. But ns 3 is not an extension of ns2, but a total new effort in building a comprehensive network simulator with new models and features that could completely replace ns 2 . Hence ns 3 is not backward compatible with ns2 [16].

ns 3 has been developed using $\mathrm{C}++$ and Python with scripting capability. ns-3 has been designed in modular fashion as a set of 
libraries. These libraries can be combined together and with other external software libraries. ns3 has the capability of being integrated with external animators and data analysis and visualization tools for better presentations of results [17].

The main shortcoming of the ns3 simulator is the lack of Graphical User Interface and lack of support for wide variety of operating systems. Currently ns 3 can be installed only on Linux operating system in the native mode and for Microsoft Windows users need to install it on Cygwin for Windows [18]. Also the non availability of backward compatibility with ns 2 will also hinder the ready acceptance of ns 3 as the default simulation tool as users will be reluctant to abandon $n s 2$ immediately.

\subsection{Experiment Setup}

Experiment setup has been created in ns 3 running on Cygwin on Windows 7. The experiment setup and the experiments carried out have been explained in detail in Section 4.

\subsection{Performance Metrics}

In order to compare the performance of the two networking architectures, it is important to select suitable set of performance metrics. Many performances metrics such as throughput, delay, delay jitter and packet delivery ratio are commonly used by researchers in quantifying the performance of their networks. Since this study is concerned with only comparing the performance of the two networking architectures, it is sufficient to use a single performance metric that can give an overall idea. Hence, in this study throughput has been selected as the metric for quantifying and comparison of the performance of the networking architecture. Throughput defined as the total number of packets received in unit time when the sending rate is kept constant. Hence throughput can be considered to be better placed than all the other metrics in quantifying the overall performance of a network.

\subsection{EXPERIMENTAL SETUP}

In order to evaluate the networking architectures a simple network architecture was created in ns3. A simple dumbbell topology with six nodes and five links with different bandwidths and delays was configured in the simulator. Figure 2 shows the experimental topology setup in ns3.

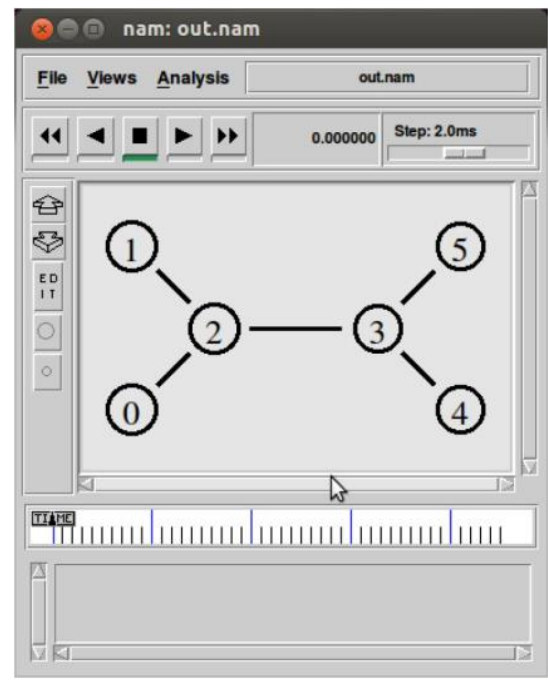

Figure 2 Network topology
Out of the six nodes in the topology, the nodes were configured as follows: two nodes (node 1 and node 6) as source nodes generating data, two nodes (node 2 and node 3 ) as bottleneck nodes and the other two nodes (node 3 and node 4 ) as consumers or sinks. The link between the nodes 2 and 3 becomes the bottleneck link as it is required to carry the information from all source.

While maintaining the same topology, the NDN architecture was introduced with certain additional functionalities as follows. The NDN topology was developed using naming schemas and trust models. Also the NDN architecture contained optional modules for special functions such as a placeholder for the data security of the code allowing users to attach as signature to the data, support for negative acknowledgements (Interest NACK) for providing fast feedback about data plane problems. It was also equipped with some additional pluggable modules such as interest rate limit, interface availability component and extensible statistics modules.

Figure 3 and 4 show the network delay and the delay jitter experienced by packets between the sender and receiver. From Figure 3, it can be seen that the packet experience larger packet delays in the end to end network compared to the NDN network. It should also be noted that the delay experienced by packets in the end to end network increases drastically in an exponential fashion when the network loading is increased. On the other hand, packets in the NDN network undergo very low packet delays the effect of increasing the network loading has marginal impact on the packet delays as shown in Figure 3.

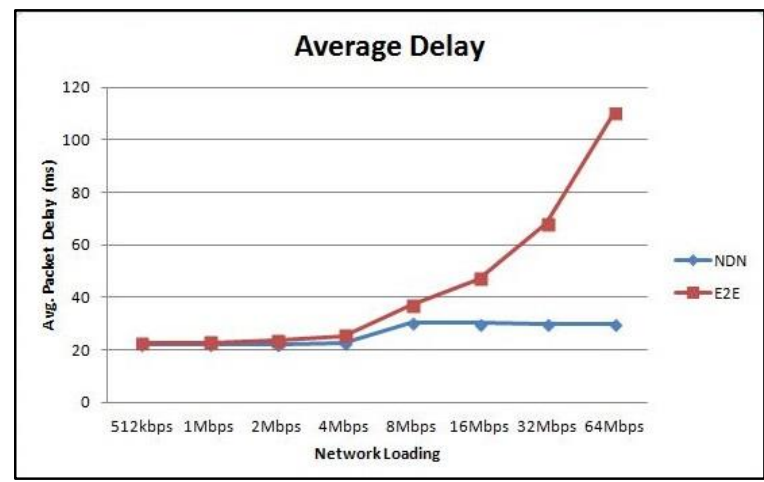

Figure 3 Packet delay under different loadings

Figure 4 shows the standard deviation of the packet delays experienced in both networks. The standard deviation of the packet delays is a measure of the delay jitter as the larger standard deviation showing larger delay jitter. The ideal situation must be zero standard deviation where all the packets undergo the same delay.

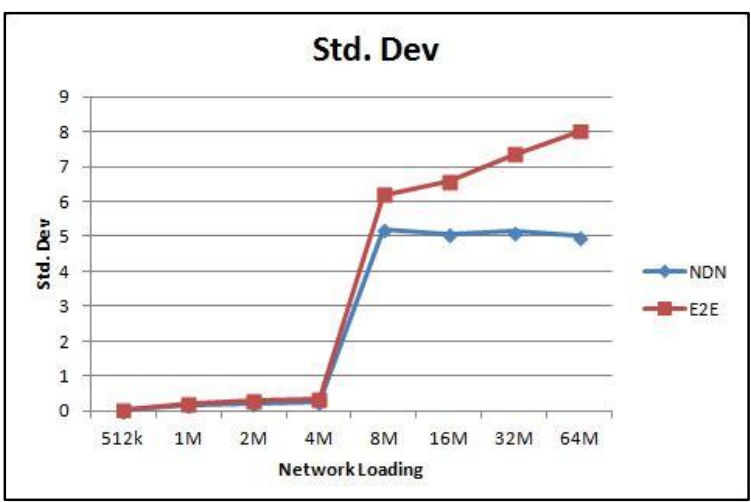

Figure 4 Standard deviation of packet delay 
From Figure 4, it can be seen that the delay jitter in the end to end network is higher than that of the NDN network. Also the delay jitter increases in the end to end network with increased loading.

The main reason for the above observation can be attributed to the basic principles and the nature of the architecture of networks themselves. In the end to end TCP/IP networks, the information packets are always requested from the source identified at the beginning of the communication itself. Hence the packets are affected by the nature and fluctuations of the intermediate network and devices. On the contrary, in the NDN network, any node who has the information can reply to any information request provided it has the information in its cache. In this experiment, all the end nodes as well as intermediate nodes (routers) are configured with the capability of holding information in their caches (in network memory). Hence a node that is closer to the destination (requester) can always answer a query reducing the network latency and resulting jitter eliminating the effect of the intermediate network to a greater extent.

Figures 5 and 6 show the effect of packet size on delay characteristics of data packets.

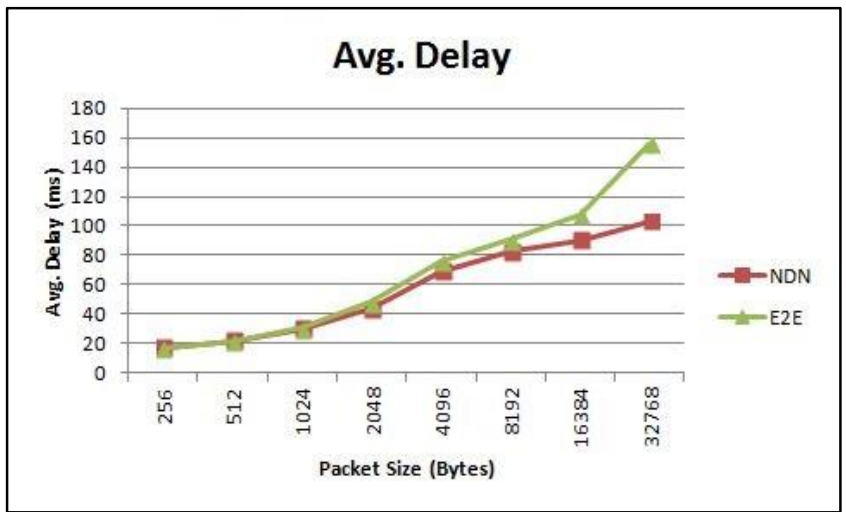

Figure 5 Packet delay vs packet size

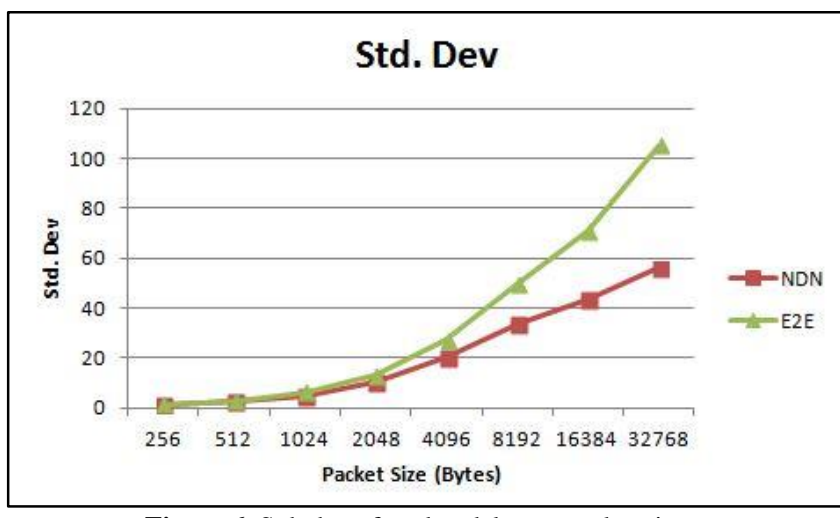

Figure 6 Std. dev of packet delay vs packet size

From Figures 5 and 6 shown above, it could be seen that in both NDN as well as end to end networks the network delay as well as delay jitter increases with that of the information packet size. This is mainly due to the fact that the network links and devices take a long a time to transmit and process larger packets compared to smaller packets. Also it could be noticed during the experiments, when the packet size was larger than a certain size, these packets underwent fragmentation resulting in a larger transmission and processing delays as all the fragments have to be received and assembled before processing them. Out of the two network paradigms, it could be noted that the end to end networks suffer more delay and jitter under both loading and increased information packet size conditions.

Figure 7 shows the throughput of the networks under various information packet sizes. From the figure it could be seen that in both networks, the throughput was $100 \%$ when the packet size was small and the throughput starts dropping when the packet size was increased. One of the main reasons for this is the fragmentation of packets. When a single fragment of a packet was not received, the entire packet was either dropped or requested for retransmission reducing the throughput of the stream. Though both networks have suffered from reduced throughput in the face of dropped packets, the end to end networks suffers more as all the packets need to be received from the source node itself. In the case of NDN, even intermediate devices actively participate in the communication using the information stored in the caches. This effectively reduces the packet losses improving the overall throughput of the network.

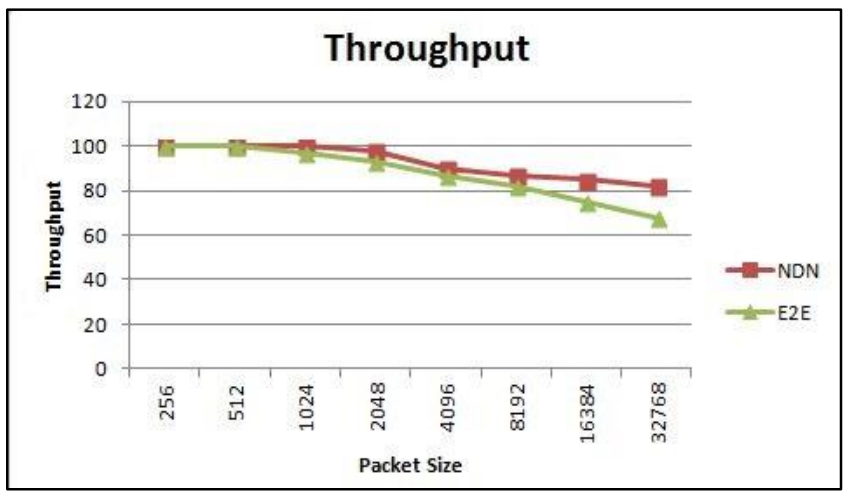

Figure 7 Throughput vs packet size

\subsection{CONCLUSIONS}

Named data networking is the newest paradigm in information networking where the content is given more prominence than the place (node) where the information is stored. NDN has many positive features compared to the traditional end to end networking based on the TCP/IP protocol. In this paper, we presented a critical analysis of both network paradigms based on their performance under various conditions. From the results obtained from the experiments, we could prove that NDN network outperforms the end to end network in all situations.

\section{References}

[1] Ghodsi, A., Shenker, S., Koponen, T., Singla, A., Raghavan, B., \& Wilcox, J. 2011. Information-centric Networking: Seeing the Forest for the Trees. 10th ACM Workshop on Hot Topics in Networks, Cambridge, MA, USA. 1-6.

[2] Ahlgren, B., Dannewitz, C., Imbrenda, C., Kutscher, D., \& Ohlman, B. 2012. A Survey of Information-Centric Networking. IEEE Communications Magazine. 50(7): 26-36.

[3] Melazzi, N. B., \& Chiariglione, L. 2013. The Potential of Information Centric Networking in Two Illustrative Use Scenarios: Mobile Video Delivery and Network Management In Disaster Situations. IEEE Communication Society Multimedia Communications Technical Committee E-Letter. 8(4): 25-28.

[4] Blumenthal, MS., \& Clark, DD. 2001. Rethinking the Design of the Internet: The End-To-End Arguments Vs. The Brave New World. ACM Transactions on Internet Technology. 1(1): 70-109.

[5] Paul, S., Pan, J., \& Jain, R. 2011. Architectures for the Future Networks and the Next Generation Internet: A Survey. Computer Communications 34: 2-42. 
[6] Jain, R. 2006. Internet 3.0: Ten Problems with Current Internet Architecture and Solutions for The Next Generation. Military Communications Conference (MILCOM 2006). Washington, DC. 1-9.

[7] Oehlmann, F. 2013. Content-centric networking. Future Internet and Innovative Internet Technologies \& Mobile Communication Seminars. Munich, Germany. 43-49.

[8] Kutscher, D., Flinck, H., \& Karl, H. 2010. Information-centric Networking-A Position Paper. 6th GI/ITG KuVS Workshop on Future Internet. Hannover, Germany. 1-2.

[9] Siris, V. A., Ververidis, C. N., Polyzos, G. C., \& Liolis, K. P. 2012 Information-Centric Networking (ICN) Architectures For Integration of Satellite Into the Future Internet. First IEEE AESS European Conference on Satellite Telecommunications (ESTEL). Rome, Italy. 1-6.

[10] Vaishnavi, V. K., \& Kuechler, W. L. 2008. Design Science Research Methods and Patterns: Improving and Innovating Information and Communication Technology. New York: Auerbach.

[11] Eker, I., \& Kara, T. Behaviour of a Water Supply System: A Modelling and Simulation Study of Activities With Some Experiments.

[12] Landsiedel, O., Wehrle, K., Titzer, BL., \& Palsberg, J. 2005. Enabling Detailed Modeling and Analysis of Sensor Networks. Praxis der Informationsverarbeitung und Kommunikation. 28(2): 101-106.
[13] Siraj, S., Gupta, AJ., \& Badgujar, R. 2012. Network Simulation Tools Survey. International Journal of Advanced Research in Computer and Communication Engineering. 1(4): 201-210.

[14] Rampfl, S. 2013. Network Simulation and Its Limitations. Seminars on Future Internet, Innovative Internet Technologies and Mobile Communications and Autonomous Communication Networks. Munich, Germany. 57-63.

[15] Saeed, T., Gill, H., Fei, Q., Zhang, Z., \& Loo, BT. 2011. An open-source and Declarative Approach Towards Teaching Large-scale Networked Systems Programming. ACM SIGCOMM Education Workshop, Toronto, Canada, 2011. 1-5.

[16] Henderson, T. R., Roy, S., Floyd S., \& Riley, G. F. 2006. ns3 Project Goals. Workshop on $n s-2$ : the IP Network Simulator (WNS2'06). Pisa, Italy. 1-8.

[17] Khan, AR., Bilal, SM., \& Othman, M. 2012. A Performance Comparison of Open Source Network Simulators for Wireless Networks. IEEE International Conference on Control System, Computing and Engineering. Penang, Malaysia. 34-38.

[18] Chhimwal, P., Rai, DS., \& Rawat, D. 2013. Comparison Between Different Wireless Sensor Simulation Tools. IOSR Journal of Electronics and Communication Engineering. 5(2): 54-60. 